\title{
What and When of Cognitive Aging
}

Timothy A. Salthouse

University of Virginia

\begin{abstract}
Adult age differences have been documented on a wide variety of cognitive variables, but the reasons for these differences are still poorly understood. In this article, I describe several findings that will need to be incorporated into eventual explanations of the phenomenon of cognitive aging. Despite common assumptions to the contrary, age-related declines in measures of cognitive functioning (a) are relatively large, (b) begin in early adulthood, (c) are evident in several different types of cognitive abilities, and (d) are not always accompanied by increases in between-person variability.
\end{abstract}

KEYWORDS_aging; cognition; reasoning; memory; speed

The phenomenon of cognitive aging has been noticed almost as long as the phenomenon of physical aging, but it is still not well understood. This is unfortunate because cognitive functioning can affect one's quality of life, and even the ability to live independently. Furthermore, cognitive functioning in early adulthood may be related to the development of pathologies such as Alzheimer's disease in later adulthood.

One way to conceptualize understanding is that it is equivalent to knowing answers to the questions of what, when, why, where, and how. In this article, I summarize some of the progress that has been achieved in describing the phenomenon of cognitive aging in terms of the questions of what and when. Although not much is yet known about why (what is ultimately responsible), where (in the nervous system), and how (via what mechanisms) age-related cognitive changes occur, a key assumption of my research is that answering these other questions will be easier as the characterization of what and when becomes more precise.

\section{WHAT AND WHEN}

It is often assumed that age-related effects on cognitive functioning are small, are limited to aspects of memory, begin relatively late in adulthood, and possibly affect only some people, so that any age-related declines are accompanied by increases in between-person variability. However, recent research in my laboratory and elsewhere suggests that these assumptions may all be incorrect. Evidence relevant to these issues can be illustrated with data aggregated across

Address correspondence to Timothy A. Salthouse, Department of Psychology, P.O. Box 400400, University of Virginia, Charlottesville, VA 22904-4400; e-mail: salthouse@virginia.edu. several recent studies in my laboratory (Salthouse, 2001a, 2001b; Salthouse, Atkinson, \& Berish, 2003; Salthouse \& Ferrer-Caja, 2003; Salthouse, Hambrick, \& McGuthry, 1998; Salthouse et al., 2000). Participants in these studies were recruited through newspaper advertisements, appeals to community groups, and referrals from other participants. Nearly all of the participants reported themselves to be in good to excellent health, and they averaged approximately 16 years of education.

Four tests were common to most of these studies. A vocabulary test involved the examinee selecting the best synonyms of target words, in each case from a set of five alternatives. A speed test required the participant to classify pairs of line patterns as the same or different as rapidly as possible. Reasoning was assessed with the Raven's Progressive Matrices, in which each test item consists of a matrix of geometric patterns with one missing cell, and the task for the participant is to select the best completion of the missing cell from a set of alternatives. Finally, a memory test involved three auditory presentations of the same list of unrelated words, with the participant instructed to recall as many words as possible after each presentation. Data for the vocabulary, speed, and reasoning tests are based on 1,424 adults, and those for the memory test are based on 997 adults.

Because the raw scores for the four tests are in different units, all of the scores have been converted to $z$ scores (by subtracting each score from the mean for that test and then dividing by the standard deviation) so that the age trends can be directly compared. The means for the $z$ scores are plotted as a function of age in Figure 1. The bars above and below each point are standard errors, which represent the precision of the estimate (i.e., the smaller the bars, the more precise the estimate). Six important observations about the data in this figure can be noted.

First, scores on the vocabulary test were higher with increased age until about the mid-50s, after which they either remained stable or declined slightly. Findings such as these have been interpreted as indicating that knowledge accumulates with increased age, but compelling explanations for why this age function is curvilinear are not yet available (Salthouse, 2003).

Second, similar negative age trends are evident in the measures of speed, reasoning, and memory. Although not represented in the figure, the correlations between age and these variables were also similar, as they were $-.47,-.48$, and -.43 , respectively, for the speed, reasoning, and memory variables.

Third, the age-related effects on the speed, reasoning, and memory variables are fairly large. Not only are the age correlations for these variables greater than most correlations involving individual differences reported in the behavioral sciences, but the average 


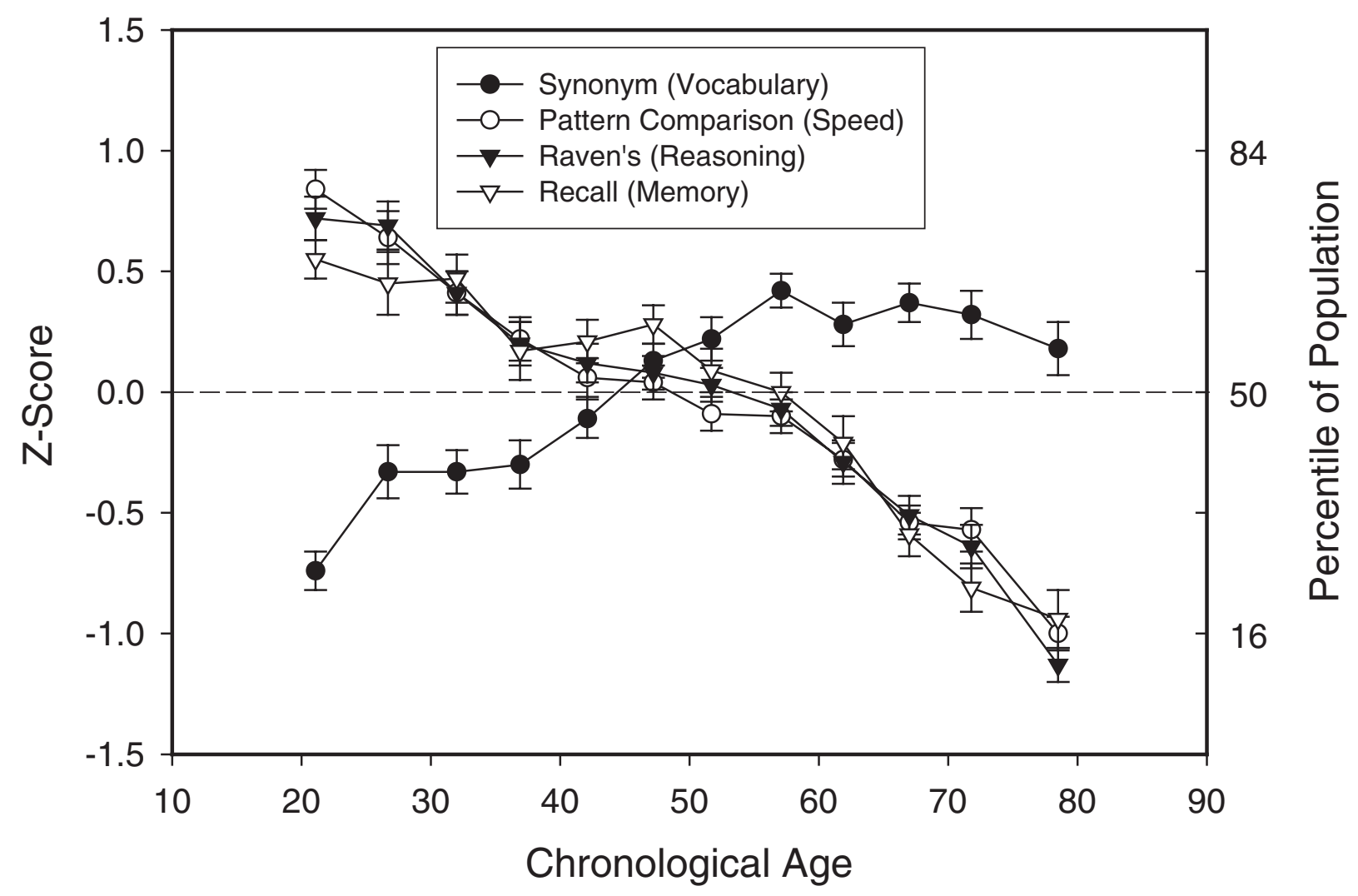

Fig. 1. Means (and standard errors) of performance in four cognitive tests as a function of age. Each data point is based on between 52 and 156 adults.

performance for adults in their early 20 s was near the 75 th percentile in the population, whereas the average for adults in their early $70 \mathrm{~s}$ was near the 20th percentile.

Fourth, the relations between age and the speed, reasoning, and memory variables are primarily linear. This observation is relevant to potential interpretations of the effects because the absence of obvious discontinuities in the functions suggests that transitions such as retirement, or menopause for women, are probably not responsible for much, if any, of the effects.

Fifth, the data in the figure indicate that age-related effects are clearly apparent before age 50 . For some variables, there may be an acceleration of the influences at older ages, but age-related differences are evident in early adulthood for each variable.

And sixth, the age-related declines in these samples are not accompanied by increases in between-person variability. One way to express the relation between age and between-person variability is in terms of the correlation between age and the between-person standard deviation for the individuals in each 5-year age group. For the data in Figure 1, these correlations were -.18 for vocabulary, -.80 for speed, -.74 for reasoning, and .13 for memory. If anything, therefore, the trend in these data is for increased age to be associated with a smaller range of scores. Instead of a pattern of increased variability that might be attributable to some people maintaining high levels of performance and others experiencing large declines, the data show a nearly constant variability that is more consistent with a downward shift of the entire distribution of speed, reasoning, and memory scores with increased age.
Many of the patterns apparent in Figure 1 have been reported in a number of individual studies (see the earlier citations), and are also evident in data from nationally representative samples used to establish norms for standardized tests such as the third edition of the Wechsler Adult Intelligence Scale (Wechsler, 1997) and the Woodcock-Johnson III Tests of Cognitive Abilities (Woodcock, McGrew, \& Mather, 2001). Results such as these suggest the following answers to the questions of the what and when of cognitive aging. With respect to what, many different types of cognitive variables are affected by increased age, and with respect to when, age-related differences appear to begin in early adulthood, probably in the $20 \mathrm{~s}$.

\section{WHY ARE THE EFFECTS NOT MORE NOTICEABLE IN EVERYDAY LIFE?}

The research I have summarized suggests that age-related cognitive declines are fairly broad, begin early in adulthood, and are cumulative across the life span. A question frequently raised when findings such as these are mentioned is, why are there not greater negative consequences of the age-related cognitive declines? I suspect that there are at least four reasons.

First, cognitive ability is only one factor contributing to successful functioning in most activities. Other factors such as motivation, persistence, and various personality characteristics are also important, and they either may be unrelated to age or may follow different age trajectories than measures of cognitive functioning. 


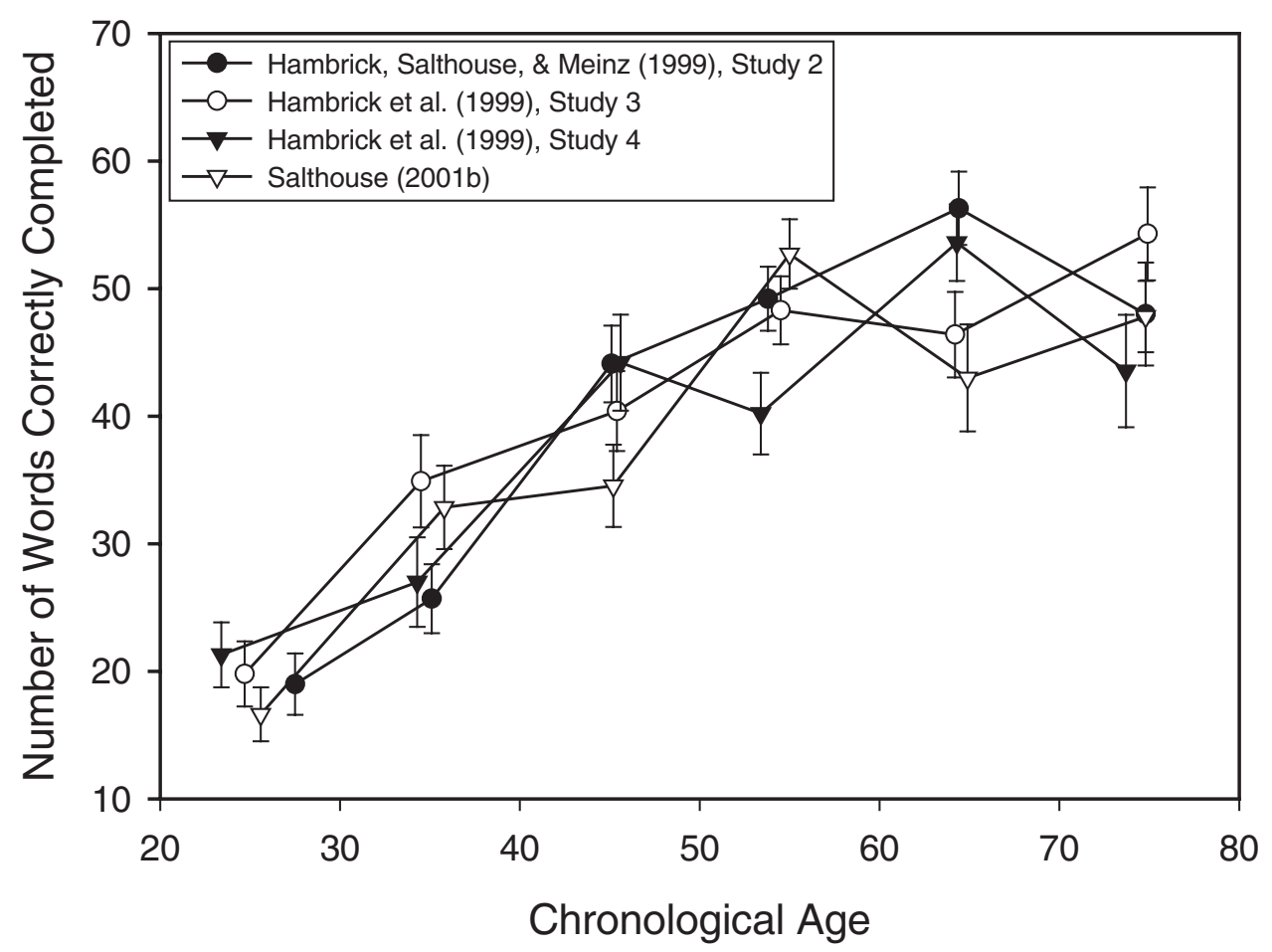

Fig. 2. Means (and standard errors) for the number of words in a New York Times crossword puzzle correctly answered in $15 \mathrm{~min}$ as a function of age. Between 195 and 218 adults participated in each study. The crossword puzzles required either 76 or 78 words for their solutions.

Second, very few situations require individuals to perform at their maximum levels because humans tend to modify their environments to reduce physical and cognitive demands. An analogy to physical ability and physical demands may be relevant here because there are well-documented age-related declines in strength, stamina, and speed, but these declines are seldom noticed in everyday life because of the relatively low physical requirements of most situations.

Third, many people may adapt to age-related changes by altering the nature and pattern of their activities. Examples of this type of adaptation are apparent in driving, because as they grow older, many adults make adjustments such as driving at different times and under different conditions, and possibly avoiding certain maneuvers, such as left turns. Accommodations such as these do not eliminate the declines, but they may serve to minimize their detrimental consequences.

And fourth, the greater experience and knowledge associated with increased age probably reduces the need for the type of novel problem solving that declines with age. Continuous age-related increases in knowledge may not be apparent in standardized tests because the tests are designed to be applicable to the general population, and much of the individual's knowledge may be increasingly idiosyncratic as he or she pursues progressively more specialized vocational and avocational interests. Nevertheless, very high levels of performance might be apparent among older adults, given the right combination of individuals and tasks. Research in my laboratory suggests, for example, that older adults demonstrate a high level of performance if they regularly work crossword puzzles and the task is solving crossword puzzles. In four recent studies, adults recruited because of their crossword-puzzle experience were asked to perform a number of activities, including spending 15 min attempting to solve a crossword puzzle taken from the New York Times. As can be seen in Figure 2, in general, the highest average level of crossword performance in every sample was achieved by adults in their $60 \mathrm{~s}$ and $70 \mathrm{~s}$.

It could be argued that successful performance in solving crossword puzzles is primarily dependent on accumulated knowledge rather than on novel problem solving or abstract reasoning. This may be the case, but I suspect that the same is true in many real-world activities. That is, much of what we typically do may be more dependent on successful access and retrieval of what we already know than on our ability to solve novel problems or reason with unfamiliar material.

\section{INVESTIGATING THE WHY, WHERE, AND HOW OF COGNITIVE AGING}

Although the phenomenon of cognitive aging is fairly well documented in terms of the questions of what and when, there is much less consensus with respect to the answers to, or even the best methods of investigating, the questions of why, where, and how. In fact, it can be argued that much of the current theoretical debate in the field of cognitive aging is not focused on distinguishing among alternative explanations, but rather is concerned with which approach is likely to be most productive in investigating causes of age-related differences in cognitive functioning. Several of the major issues can be described in terms of the following dichotomies, although it should be recognized that these are simplifications, and that this list is by no means exhaustive.

\section{Micro Versus Macro}

One theoretical issue is whether the primary focus should be on determining which specific aspects (e.g., theoretical processes or com- 
ponents) of cognition are most (or least) affected by aging, or whether several variables should be examined simultaneously to determine the extent to which the age-related effects on a particular variable are unique to that variable or are shared with other variables. Advocates of the former, micro, perspective point out that most cognitive tasks can be assumed to involve multiple processes, and thus an overall measure of performance in nearly any task is likely to represent an unknown mixture of theoretically distinct processes that may be difficult to interpret. Advocates of the latter, macro, perspective emphasize that a large number of cognitive variables have been found to be related to age, and that analyses have revealed that agerelated influences on different types of cognitive variables are not statistically independent of one another. Researchers favoring the macro perspective have therefore argued that the age-related effects on particular cognitive tasks may be symptoms of a broader phenomenon, and consequently that it may not be very meaningful to attempt to provide a distinct explanation for the age differences in each variable.

\section{Proximal Versus Distal}

A second theoretical issue is whether researchers should concentrate on specifying characteristics (e.g., strategy, efficiency of specific hypothesized processes, adherence to particular sets of beliefs) associated with the performance differences of adults of different ages at the time of assessment (proximal factors), or whether researchers should try to identify factors occurring earlier in life that may have contributed to any differences observed at the current time (distal factors). The key question in this connection is whether it is more important to specify precisely how the performance of people of different ages differs at the current time or to investigate the role of earlier life experiences in producing those differences.

\section{Moderation Versus Manipulation}

A third issue relevant to the investigation of causes of cognitive aging arises from the fact that true experiments are not possible because the critical variable of age cannot be randomly assigned. Researchers differ in which of two approximations to true experiments they think will be most fruitful. On one side are those who say the focus should be on determining if particular characteristics (moderators) are associated with differences in the age-related trends on various cognitive variables. Their approach is to compare the age-related trends of preexisting groups (e.g., people sharing various lifestyle characteristics). On the other side are researchers who say that the most will be learned by attempting to alter people's current level of performance by some type of intervention. Using this approach, they hope to identify manipulations that influence the relation between age and level of performance.

\section{Difference Versus Change}

One of the perennial issues in developmental research is whether the results of cross-sectional comparisons can be considered informative about age-related changes, or whether all inferences about aging must be based on directly observed longitudinal changes. There is little dispute that people of different ages who are tested at the same point in time may also differ in other characteristics, and thus results of cross-sectional comparisons might not directly reflect effects of aging. There is also considerable agreement that there are several possible influences on longitudinal changes, including effects related to practice or learning from one occasion to the next and effects associated with changes in the society or culture in which the individual lives. However, there is much less consensus about the best method of distinguishing between "age" and "nonage" influences in each type of design. On the one hand, researchers favoring cross-sectional methods feel that it is plausible to assume that people who are of different ages and observed at the same point in time were similar in most important respects when they were at the same age, so that it is reasonable to make inferences about maturational changes on the basis of crosssectional differences. On the other hand, researchers favoring longitudinal methods frequently assume that the maturational component of change can be distinguished from other components of change, such as practice effects and effects of sociocultural change, either because the latter are small relative to maturational effects or because they can be separated by statistical or other means.

It is probably healthy for a field to pursue different approaches to explanation when the level of understanding is relatively limited. However, it is probably also the case that progress toward answering the why, where, and how of cognitive aging will not be reached until there is some agreement among different theoretical perspectives on the best methods of addressing those questions.

\section{CONCLUSION}

To summarize, recent research in my laboratory and elsewhere has provided considerable information about the what and when of cognitive aging. We are also beginning to learn about the implications of this phenomenon for functioning outside of the research laboratory, but, perhaps because of different perspectives on the best methods of investigation, much less is currently known about the why, where, and how of this phenomenon.

\section{Recommended Reading}

Craik, F.I.M., \& Salthouse, T.A. (2000). Handbook of aging and cognition (2nd ed.). Mahwah, NJ: Erlbaum.

Salthouse, T.A. (1991). Theoretical perspectives in cognitive aging. Mahwah, NJ: Erlbaum.

Acknowledgments - This research was supported by National Institute on Aging Grants AG06826 and AG19627 to the author.

\section{REFERENCES}

Hambrick, D.Z., Salthouse, T.A., \& Meinz, E.J. (1999). Predictors of crossword puzzle proficiency and moderators of age-cognition relations. Journal of Experimental Psychology: General, 128, 131-164.

Salthouse, T.A. (2001a). Attempted decomposition of age-related influences on two tests of reasoning. Psychology and Aging, 16, 251-263.

Salthouse, T.A. (2001b). Structural models of the relations between age and measures of cognitive functioning. Intelligence, 29, 93-115. 
Salthouse, T.A. (2003). Interrelations of aging, knowledge, and cognitive performance. In U. Staudinger \& U. Lindenberger (Eds.), Understanding human development: Lifespan psychology in exchange with other disciplines (pp. 265-287). Berlin, Germany: Kluwer Academic.

Salthouse, T.A., Atkinson, T.M., \& Berish, D.E. (2003). Executive functioning as a potential mediator of age-related cognitive decline in normal adults. Journal of Experimental Psychology: General, 132, 566-594.

Salthouse, T.A., \& Ferrer-Caja, E. (2003). What needs to be explained to account for age-related effects on multiple cognitive variables? Psychology and Aging, 18, 91-110.
Salthouse, T.A., Hambrick, D.Z., \& McGuthry, K.E. (1998). Shared age-related influences on cognitive and non-cognitive variables. Psychology and Aging, 13, 486-500.

Salthouse, T.A., Toth, J., Daniels, K., Parks, C., Pak, R., Wolbrette, M., \& Hocking, K. (2000). Effects of aging on the efficiency of task switching in a variant of the Trail Making Test. Neuropsychology, 14, 102-111.

Wechsler, D. (1997). Wechsler Adult Intelligence Scale-third edition. San Antonio, TX: Psychological Corp.

Woodcock, R.W., McGrew, K.S., \& Mather, N. (2001). Woodcock-Johnson III Tests of Cognitive Abilities. Itasca, IL: Riverside. 